\title{
Activity levels for female students at Qassim University
}

\section{Alotaibi, Abdulazeem Saud $\mathbf{S}^{1}$}

\section{Introduction:}

Physical inactivity is a common phenomenon in modern societies, leading to a lack of concentration, low energy level, chronic fatigue syndrome and chronic diseases such as, anxiety and depression, ischemic heart diseases, diabetes and even cancer (Newton et al., 2011; Lavie et al., 2015; Wisloff and Lavie, 2017).

It is widely accepted that engaging in regular physical activity is an effective preventative measure to reduce the risk of developing a variety of diseases improved general health and well-being as well as major impacts on mental health and productivity (Joseph et al., 2014; AlHazzaa et al., 2011; Tremblay et al., 2011; WHO,2019). In Oldridge (2008) western countries such as the United Kingdom, Switzerland and the United States, 3\% of total direct health finance resources are used directly to tackle or prevent health issues related to physical inactivity. In addition, nearly $7 \%$ of Australia's health burden has been attributed to physical inactivity (Begg et al., 2007).

In the Kingdom of Saudi Arabia (KSA), such as in other wealthy countries, inactivity has been at the forefront of health and wellbeing discussions in recent times. In the past decade the physical activity and fitness of Saudi Arabia's population has decreased dramatically. The hot desert climate, a lack of indoor facilities and affordable exercise venues, as well as cultural beliefs have all contributed to this phenomenon (Donnelly et al., 2018). Three quarters of the adult population are classified as inactive in KSA (Al-Hazzaa, et al., 2011). Nevertheless, this sedentary lifestyle has been associated with a greater risk of early mortality from obesity related diseases that equate to $6 \%$ of the total death rate globally each year (Henriksen et al., 2019).

\footnotetext{
1 Associate Professor and Head of physical education department at Qassim University Email. Athom_14@hotmail.com
} 
Strong evidence directly links some chronic diseases to physical activity levels such as diabetics and obesity, both of which are a major concern in the KSA (Mabry et al., 2016). This phenomenon is shared by most wealthy developed countries not just KSA. The World Health Organization reported that $81 \%$ of the age group 11-17 years were not fulfilling the WHO activity guideline of 30 mins daily exercise (WHO, 2019).

There are few studies that have investigated the level of physical activity among KSA women. A study conducted by Al-Eisa et al. (2016) showed that the KSA adolescent women exhibit a very poor level of activity. Alarmingly, the level of activity among adolescent women is classified as the same level expected for women over 65 years old. Most studies that investigated the level of activity among KSA women have used quantitative data that is limited to within large cities. Cultural differences and expectations differ between women's attitudes to exercise and physical health in larger urban areas compared to in more rural environments, with urban areas having a greater access to exercise facilities also.

The aim of this study is to assess the level of inactivity for females within the student population of al-Quassim University, a more rural setting than most previous studies in KSA, compared to the international daily activity requirement specified by the WHO. An exploration of potential interventions aimed at increasing levels of physical activity is addressed to reduce inactivity amongst the female population.

\section{Materials and method}

This study was approved by the University Ethics Committee and was conducted in accordance with the guidelines of the declaration of Helsinki. Anthropometric measurements were taken prior to the trial including height and mass. This study has been conducted at Al-Qassim University (KSA), 127 female students from Al-Qassim university participated in this study (Age $20 \pm 1$ year, Mass $55.63 \pm 9.91 \mathrm{~kg}$, Stature $157.18 \pm 5.25 \mathrm{~cm}$ ). This equates to $40 \%$ of the 
overall female participants enrolled for the 2017-2018 academic year. Smart wristband bracelet (Xiaomi MGW4024GL Mi Band 2 \& China) was used to track daily step counts, walking distance, level of activity, amount of sleep, resting heart rate and the daily number of calories burned. The app of Mi Fit has been used to synchronise the data collected from the smart wrist band.

All participants where taught how to use the device accurately in a pilot study prior to the start of the main study. Participants were asked to wear the wrist band for 7 days for the full 24hour time period. For this study, the participants were divided into two groups: inactive group and active group.

The reliability and validity of the wrist band used in this study (Xiaomi MGW4024GL Mi Band 2 \& China) has been compared and tested against the Polar M430 (Polar Electro Oy, Kempele, Finland) with no significance difference in performance capabilities or reliability between Mi Band and Polar M430 $(\mathrm{P}<0.00)$

\section{Statistical Analysis}

Data were analysed using a two-way repeated measure ANOVA with Bonferroni-adjusted, post hoc tests to determine which factor(s) are affecting inactivity among the participants using SPSS20 Statistical Software, IBM.UK. This analysis revealed the global effect of sleep duration, distance covered, calories burned, heart rate and the inactivity level. A paired sample T-test was used to determine differences between active and inactive measured variables. Statistical significance was accepted at $\mathrm{P}<0.05$. Results are represented as mean values \pm standard deviation (SD).

\section{Results:}


There was a significant difference between active and inactive female participants on all measured variables shown in table $1(\mathrm{p}=0.01)$. Active female participants showed $350 \%$ higher daily steps compared to inactive female participants ( $\mathrm{p}=0.01$, Cohen's $d$ for this test was 0.83$)$. Hence, the level of activity has affected positively the resting heart rate and sleeping hours on active females ( $\mathrm{p}=0.00$, Cohen's $d 0.76$ and $\mathrm{p}=0.01$, Cohen $d 0.69$ respectively). For active participants twice the daily calories where burned compared to the inactive participation group ( $\mathrm{p}=0.00$ Cohen $d 0.71)$.

Table 1: The difference between active and inactive measured variables

\begin{tabular}{|l|l|l|}
\hline & Active female & Inactive female \\
\hline Daily steps (No.) & $5475.2 \pm 352^{*}$ & $1559.5 \pm 154.6^{*}$ \\
\hline Calories burned (Cal.) & $2508 \pm 157.6^{*}$ & $1150.6 \pm 128.9^{*}$ \\
\hline Sleeping (Hrs) & $8.6 \pm 2.6^{*}$ & $7.6 \pm 3.6^{*}$ \\
\hline Resting HR (beats/min) & $76.5 \pm 13.6^{*}$ & $82.5 \pm 19.7^{*}$ \\
\hline BMI & & $22.3 \pm 3.84$ \\
\hline
\end{tabular}

‘*' symbol denotes a significant difference.

The results show a positive correlation between the number of strides covered by participants and the amounts of calories burned. While a negative significant relation between steps covered and number of sleeping hours (Table 2).

Table 2: Correlation between steps number, HR, sleep and calories consumed

\begin{tabular}{|l|l|l|l|l|}
\hline \multicolumn{6}{|c|}{ Correlation between steps number, HR, sleep and calories consumed } \\
\hline Variables & Calories & Sleep & HR & Mass \\
\hline
\end{tabular}




\begin{tabular}{|l|l|l|l|l|l|}
\hline Steps (No.) & Correlation & $0.288^{* *}$ & $-0.184^{*}$ & -0.46 & -0.192 \\
\cline { 2 - 6 } & P values & 0.002 & 0.038 & 0.612 & 0.34 \\
\hline
\end{tabular}

‘*' symbol denotes a significant difference. ' $*$ ' $=0.05$ confidence interval and '**' denotes 0.005 confidence interval

\section{Discussion:}

The findings show that the recommended daily activity by WHO are not fulfilled by both groups who took part in this study. Hence, this study adds to the existing literature that inactivity can be one of the major economic burdens in modernised KSA and that this sedentary lifestyle is becoming a significant public health issue in the Kingdom. Therefore, it appears to be increasingly widespread in many remote counties in the kingdom despite being linked to a range of chronic health conditions. Inactive female participants showed a significant sedentary life style compared to their comrade at the same university. The female level of inactivity in this study is represented by the number of daily steps, which is representing only $15 \%$ from the daily recommended by WHO. Nevertheless, the active participants as well fail by $50 \%$ to fulfil the WHO recommendation. To put it in context, according to 10000 daily steps recommendation by $\mathrm{WHO}, 100 \%$ from the present study subjects are classified as inactive population. Although, the BMI of the two groups are restricted to the healthy limit and it is intuitive that the level of the daily calories is correlated with the level of activity among our participants.

The result of the currents study supports the view the level of activity declines each year in KSA women. Female today is KSA expend $320 \%$ less steps than did their counterparts of 8 years ago (Al-Eisa et al., 2016).

There are several behaviours has leading to the increase the level of inactivity includes working at a computer, television viewing, reading, talking with friends in person or on the telephone 
as well as the childhood attitudes to exericse and cultural differences between urban area and cities in KSA. In addition, to the best to our knowledge there is a direct effect of sedentary childhood life compared on the later adolescent life in term of physical activity. Thus, there is scare data in KSA regarding this matter, however, children in modern life expend 400 times less energy than did their counterparts of their parents at their age and are $40 \%$ less active than they were 40 years ago (Mavrvouniotis, 2012). In KSA a range of 43\%-99\% of inactivity among the population has been reported (Al-Eisa et al., 2016).

Moreover, the lack of motivation to exericse due to the combination of physical obstacles, social barriers and the hot weather discourages outdoors activities. Parental preferences favour spiritual and educational statue over physical activities and therefore contribute to reduced physical activity whilst simultaneously encouraging sedentary lifestyle. In addition, according to WHO Arab females were more than double the inactivity rate than non-Arabs. This physically inactivity among Arab females has been linked to many factors such as tiredness, lack of social support, a culturally-restrictive sex role and behavioural expectations for women, lack of facilities or governmental support to fund female sport (Benjamin and Donnelly, 2013).

The outcome of our study supports in the findings of Mabry et al. (2009) where three quarters of the regional female population are classified as inactive. Our results are highly concerning due to the known links between inactivity and health problems such as cardiovascular and respiratory events. Moreover, morbidities associated with physical inactivity are the two leading causes of death in the KSA.

The author perceives great difficulty in achieving a minimum threshold of 30 minutes of moderate to high intensity daily exercise without governmental support. Encouraging sport and higher physical activity within the female population will prevent a range of health 
complications as outlined above and ultimately put extra strain on the national healthcare system.

There is debate amongst current research as to the threshold minimum daily activity level required for a healthy lifestyle. Warburton and Bredin's, (2016) research suggest

that the threshold of exercising 30 mins a day actually does not seem to exist as this idea of 150 mins weekly moderate to vigorous physical activity become a barrier to healthy living for those who could benefit from simply becoming more active at lower levels (Warburton and Bredin, 2016). Thus, even small quantities of exericse has been reported to reduce premature mortality as well as the primary and secondary prevention various chronic diseases such as cancer, diabetes and cardiovascular conditions (Warburton and Bredin, 2016; Wen et al., 2011). Furthermore, the set of a regulation by the local authorities which compel the female participants to take part in the daily physical activity program could be a virtuous idea.

Overall, in order to increase physical activity participation among female participants at Qassim university participants aiming at chronic disease prevention and healthy life, the optimal strategy should make use of a number of proven approaches that are grounded in relevant theories and targeting the individual and their environment.

\section{Conflicts of Interest}

The authors of this publication had research support and was sponsored by Qassim University. The authors were wholly responsible for the research conducted in this paper and present the work without conflict of interest. 


\section{Reference:}

Al-Hazzaa, H. M., Abahussain, N. A., Al-Sobayel, H. I., Qahwaji, D. M., \& Musaiger, A. O. Physical activity, sedentary behaviors and dietary habits among Saudi adolescents relative to age, gender and region. International Journal of Behavioral Nutrition and Physical Activity, 2011; 8: (1), 140.

Al-Eisa, E., Al-Rushud, A., Alghadir, A., Anwer, S., Al-Harbi, B., Al-Sughaier, N., et al. Effect of motivation by "Instagram" on adherence to physical activity among female college students. BioMed research international, 2016;6.

Henriksen, A., Grimsgaard, S., Horsch, A., Hartvigsen, G., \& Hopstock, L. Validity of the Polar M430 Activity Monitor in Free-Living Conditions: Validation Study. JMIR formative research. 2019; 3(3): e14438.

J.L. Newton, J. Pairman, K. Hallsworth, S. Moore, T. Plötz, M.I. Trenell, Physical activity intensity but not sedentary activity is reduced in chronic fatigue syndrome and is associated with autonomic regulation, QJM: An International Journal of Medicine. 2011;104: 681-687.

Lavie, C. J., Arena, R., Swift, D. L., Johannsen, N. M., Sui, X., Lee, et al. Exercise and the cardiovascular system: clinical science and cardiovascular outcomes. Circulation research. 2015; 117:(2), 207-219.

Tremblay MS, Warburton DER, Janssen I, Paterson DH, Latimer AE, Rhodes RE, et al. New Canadian physical activity guidelines. Appl. Physiol. Nutr. Metab. 2011;36(1): 36-46

Oldridge NB. Economic burden of physical inactivity: healthcare costs associated with cardiovascular disease. European Journal of Cardiovascular Prevention \& Rehabilitation. 2008;15(2):130-139.

Begg S, Vos T, Barker B, Stevenson C, Stanley L, Lopez AD. In: Volume PHE 82. AIHW C, editor. 2007. The burden of disease and injury in Australia 2003.

Donnelly TT, Al-Thani A-AbM, Benjamin K, Al-Khater A-H, Fung TS, Ahmedna M, et al. Arab female and male perceptions of factors facilitating and inhibiting their physical activity: Findings from a qualitative study in the Middle East. PLoS ONE. 2018; 13(7): e0199336.

Mavrovouniotis, F. Inactivity in Childhood and Adolescence: A Modern Lifestyle Associated with Adverse Health Consequences. Sport Science Review. 2012; 3-4.

Benjamin, K Donnelly, T.T. Barriers and facilitators influencing the physical activity of Arabic adults: a literature review. Avicenna. 2013;8. 
Mabry, R.M., Reeves, M.M., Eaking, E.G., Owen, N. Evidence of physical activity participation among men and women in the countries of the Gulf Cooperation Council: a review. Obes. Rev. 2009; 11: 457-464

Joseph, R. P., Royse, K. E., Benitez, T. J., \& Pekmezi, D. W. Physical activity and quality of life among university students: exploring self-efficacy, self-esteem, and affect as potential mediators. Quality of life research. 2014; 23: 659-667.

Warburton D.E., Bredin S.S. Reflections on Physical Activity and Health: What Should We Recommend? Can. J. Cardiol. 2016;32:495-504.

Wen C.P., Wai J.P.M., Tsai M.K., Yang Y.C., Cheng T.Y.D., Lee M.-C., Chan H.T., Tsao C.K., Tsai S.P., Wu X. Minimum amount of physical activity for reduced mortality and extended life expectancy: A prospective cohort study. Lancet. 2011;378:1244-1253.

Wisloff, U., Lavie, C. J. Taking Physical Activity, Exercise, and Fitness to a Higher Level. Progress in cardiovascular diseases. 2017;60:(1), 1.

Organization, W. H. (2019). Global action plan on physical activity 2018-2030: more active people for a healthier world: World Health Organization. 